in vivo $34: 2113-2118(2020)$

doi:10.21873/invivo.12016

\title{
A Monocentric Retrospective Observational Study of Comorbidities in Patients Affected by Autoimmune Bullous Diseases
}

\author{
MARTINA FERRANTI, GIULIA GOBBO, GIULIA TADIOTTO CICOGNA and MAURO ALAIBAC
}

Unit of Dermatology, University of Padua, Padua, Italy

\begin{abstract}
Background/Aim: Autoimmune bullous diseases (AIBDs) of the skin and mucosae include a heterogeneous group of chronic diseases, which could be associated with various comorbidities. The purpose of this study was to evaluate the comorbidity profiles of patients affected by $A I B D s$, who referred to the Dermatological Clinic of Padua from December 2015 to June 2018. Patients and Methods: A monocentric retrospective observational study was conducted on 157 patients with diagnosis of AIBDs. Patients' comorbidities were investigated during the periodic visits of follow-up and through the analysis of computerized medical records. Results: Among the 157 patients, 40 (25.5\%) were diagnosed with PV, 15 (9.6\%) with PF, and 102 (64.9\%) with $B P$. Nine different comorbidities were observed, but only two of these were statistically significantly associated with BP: type 2 diabetes $(p=0.0142)$ and neuropsychiatric disorders $(p=0.015)$. Conclusion: $B P$ is statistically significantly associated with type 2 diabetes mellitus and neuropsychiatric diseases. The correlation with neuropsychiatric pathologies is interesting for the possible bidirectional role in their etiology. The association with type 2 diabetes mellitus could suggest more caution in the administration of systemic corticosteroids, especially in elderly patients.
\end{abstract}

The high prevalence of autoimmune bullous diseases (AIBDs) among elderly and multimorbid patients, prompted several authors to study the link of AIBDs with other diseases.

This article is freely accessible online.

Correspondence to: Mauro Alaibac, MD, Ph.D., Unit of Dermatology, University of Padua, Via Gallucci 4, 35128 Padua, Italy. Tel: +39 0498212901, Fax +0498218891, e-mail: mauro.alaibac@unipd.it

Key Words: AIBDs, autoimmune bullous diseases, bullous pemphigoid, pemphigus, comorbidities, type 2 diabetes mellitus, neuropsychiatric diseases.
Diabetes mellitus, hypertension, infections, neoplasms, cardiovascular diseases, neuropsychiatric disorders and other autoimmune diseases are the comorbidities most frequently linked to AIBDs, although the most significant associations have been observed in patients with bullous pemphigoid (BP) (1).

The presence of comorbidities in patients with AIBDs is relevant not only for the outcome, but also for the overall evaluation of the therapy and patient management. Indeed, comorbidities such as diabetes mellitus, hypertension, chronic infections and malignancies must be considered when systemic therapies are evaluated. For example, patients whose comorbidities preclude systemic corticosteroids (i.e. elderly patients with uncontrolled diabetes or hypertension), should be treated with topical drugs or steroid sparing agents, like antibiotics or systemic immunosuppressants (2).

Another issue concerning the comorbidities, is their correlation or not with the therapies previously administered to treat the AIBDs: in different studies, type 2 diabetes, hyperlipidemia, hypertension, infections, osteoporosis and adrenal insufficiency, were related to the specific therapy chosen to treat these patients (3). In particular, a strong correlation has been observed between systemic corticosteroids and type 2 diabetes, hypertension, osteoporosis and insomnia (4).

\section{Patients and Methods}

Trial design. A monocentric retrospective observational study was conducted on 157 patients affected by AIBDs who referred to the Dermatological Clinic of Padua from December 2015 to June 2018. Investigation on the comorbidities was realized through the analysis of computerized medical records or by directly interviewing patients during their periodic follow-up at the Dermatological Clinic.

The following data were recorded: age at diagnosis, sex, therapy and concomitant medications, comorbidities (neurological and psychiatric disorders, hypertension, diabetes mellitus, thyroid dysfunction, psoriasis, history of neoplasms and venous thromboembolism, other skin and mucosal diseases).

The diagnosis of additional diseases was based on a reliable medical history or medical documentation that included the appropriate test results. Patients allowed the collection of further information 
regarding the evolution of their AIBD, such as the number of remissions and exacerbations, the QOL (quality of life) and the adverse effects due to the dermatological therapy.

Statistical analysis. For the statistical analysis, comorbidities were grouped as follows:

- Type 2 diabetes mellitus;

- Hypertension;

- Heart disease (for example, coronary heart disease, cardiac arrhythmia and congestive heart failure);

- Neuropsychiatric diseases [dementia, stroke, Parkinson's disease, Alzheimer's disease, psychosis, anxiety, depression, epilepsy and amyotrophic lateral sclerosis (ALS)];

- Malignancies;

- Autoimmune diseases (Hashimoto's thyroiditis, Basedow-Graves' disease, rheumatoid arthritis, psoriasis, type 1 diabetes mellitus, Crohn's disease);

- Obstructive respiratory diseases [asthma, chronic obstructive pulmonary disease (COPD)];

- Osteoporosis;

- Kidney disease (e.g., chronic kidney disease, lupus nephritis and nephrotic syndrome);

The association between comorbidities and AIBDs was evaluated with the Fisher exact test.

The results of the analysis are expressed as the absolute number and the relative percentage of subjects of each AIBD affected by a specific comorbidity, and the value of statistical significance " $p$ " ( $p$ value, probability value). The results were considered statistically significant when $p<0.05$.

The data were analyzed with the SAS 9.4 program (SAS Institute Inc., Cary, NC, USA) for Windows.

\section{Results}

In this study, 157 patients were included: 102 (64.9\%) with BP, 40 (25.5\%) with pemphigus vulgaris (PV) and 15 (9.6\%) with pemphigus foliaceus $(\mathrm{PF})$.

In the subgroup of BP there were $46(45.1 \%)$ males and $56(54.9 \%)$ females. The mean age was 77.2 years (range $=26-98$ years) and the majority $(88.2 \%$ ) was older than 60 years old.

In the subgroup of PV, the number of males and females was equivalent $(20: 20)$. The mean age was 62 years (range $=34-85$ years).

In the subgroup of PF, there were 9 females $(60 \%)$ and 6 males $(40 \%)$. The mean age was 68.1 years (range $=46-88$ years).

Thus, PV and PF patients were younger than BP patients (mean age of 62 years and 68.1 years versus 77.2 years, respectively).

Nine different comorbidities were observed: type 2 diabetes mellitus, hypertension, cardiovascular diseases, neuropsychiatric disorders, malignancies, autoimmune diseases, obstructive respiratory diseases, osteoporosis and kidney diseases (Table I).

Because of the low number of patients with PV and PF, statistical analysis was performed only for patients with BP. Two comorbidities were significantly associated with BP: type 2 diabetes mellitus $(14.7 \% ; p=0.0142)$ and neuropsychiatric disorders $(19.6 \% ; p=0.015)$ (Table II).

With regard to the association between AIBDs and neuropsychiatric disorders, $37.5 \%$ of patients (9/24) were affected by mood disorders or psychosis, and used antidepressant drugs (i.e. trazodone, paroxetine, duloxetine and venlafaxine), antipsychotics (i.e. valproate, quetiapine, haloperidol) and benzodiazepines.

The mean age of patients with $\mathrm{BP}$ and neurological diseases was higher than the mean age of patients with BP without neurological diseases (83.4 years versus 75.9 years, respectively). The most frequent neurological comorbidities in BP patients were mood disorders (8 patients, $7.84 \%$ ), followed by epilepsy (3 patients, $2.94 \%$ ), Parkinson's disease (2 patients, 1.96\%), Alzheimer's disease (2 patients, $1.96 \%$ ) and stroke (2 patients, $1.96 \%$ ).

With regard to the association between type 2 diabetes mellitus and BP, the possible role of DPP-4 inhibitors in the onset of the BP was studied in detail. Among the 102 patients with BP, $15(14.71 \%)$ had a previous diagnosis of type 2 diabetes mellitus, of whom $5(4.9 \%)$ were treated with DPP-4 inhibitors, in particular linagliptin (3 patients) and vildagliptin (2 patients).

\section{Discussion}

Among the 157 selected patients of our study diagnosed of AIBDs, 64.9\% (102/157) were affected by BP, $25.5 \%$ $(40 / 157)$ by PV and $9.6 \%(15 / 157)$ by PF. Results were obtained only for patients with BP, given the limitations deriving from a small sample size of patients with PV and PF. About the nine comorbidities detected, we observed several patients with different autoimmune disorders, hypertension, cardiovascular diseases and neoplasms, but only type 2 diabetes mellitus and neuropsychiatric diseases were reported as significantly associated with BP.

Bullous pemphigoid is the most common form of AIBDs and affects a range of the population generally older than the other AIBDs. In these patients, old age and immunosuppressive therapy are important triggering factors in the onset of new pathologies or in the worsening of pre-existing pathologies. In particular, cardiovascular diseases result the most frequent comorbidities (70\%), followed by CNS disorders (37\%), type 2 diabetes (15\%) and malignant tumors (14\%) (5). In their study, Kremer et al. have shown that arterial hypertension was the most frequent comorbidity in patients with BP (64\% of patients, $p=0.04)$ (6). Conversely, the use of some antihypertensive drugs (e.g. loop diuretics) was described as a possible triggering factor in the pathogenesis of BP (7). Several theories have been proposed regarding the link between drugs and the development of BP: drugs could act as haptens or could cause a dysregulation of the immune system modifying the function of suppressor T-cells (8). Furthermore, BP, like 
Table I. Comorbidities observed in the 157 patients affected by autoimmune bullous diseases (AIBDs), grouped in (PV) pemphigus vulgaris, pemphigus foliaceus $(P F)$ and bullous pemphigoied (BP).

\begin{tabular}{lcccc}
\hline Comorbidities in AIBDs & $\mathrm{PV}\left(\mathrm{n}^{\circ}, \%\right)$ & $\mathrm{PF}\left(\mathrm{n}^{\circ}, \%\right)$ & $\mathrm{BP}\left(\mathrm{n}^{\circ}, \%\right)$ & Total $\left(\mathrm{n}^{\circ}, \%\right)$ \\
\hline Type 2 diabetes mellitus & 0 & $2(13.3 \%)$ & $15(14.7 \%)$ & $17(10.8 \%)$ \\
Hypertension & $11(27.5 \%)$ & $2(13.3 \%)$ & $29(28.4 \%)$ & $42(26.7 \%)$ \\
Cardiovascular diseases & $10(25 \%)$ & $6(40 \%)$ & $17(16.6 \%)$ & $33(21 \%)$ \\
Neuropsychiatric disorders & $1(2.5 \%)$ & $3(20 \%)$ & $20(19.6 \%)$ & $24(15.2 \%)$ \\
Malignancies & $4(10 \%)$ & $1(6.67 \%)$ & $8(8.8 \%)$ & $13(8.2 \%)$ \\
Autoimmune diseases & $7(17.5 \%)$ & $1(6.67 \%)$ & $16(15.6 \%)$ & $24(15.2 \%)$ \\
Obstructive respiratory diseases & $2(5 \%)$ & 0 & $2(1.9 \%)$ & $4(2.5 \%)$ \\
Osteoporosis & $2(5 \%)$ & 0 & $7(5.8 \%)$ & $8(5 \%)$ \\
Kidney diseases & 0 & 0 & $7(6.8 \%)$ & $7(4.4 \%)$ \\
\hline
\end{tabular}

other autoimmune diseases, is linked with an increased risk of venous thromboembolism (9-15). Chronic inflammation, through the production of pro-inflammatory cytokines, is the key element in perpetuating endothelial dysfunction, stimulating the coagulation cascade and promoting a thrombophilic state (16-19). In the light of these observations, a prophylactic anticoagulation therapy, especially in patients with other pro-thrombotic risk factors, may be indicated (9).

Our study also indicated that there is a statistically significant correlation between BP and type 2 diabetes mellitus $(p=0.0142)$ : among the 102 patients with BP, 15 $(14.71 \%)$ had a previous or subsequent diagnosis of type 2 diabetes mellitus. The cases in which BP preceded the diagnosis of type 2 diabetes mellitus may be related to the treatment with systemic corticosteroids, to which the majority of these patients are subjected. Several studies have shown that patients with BP treated with systemic corticosteroids had more complications than those treated with topical corticosteroids (20). Furthermore, it has been observed that the incidence of serious side effects (e.g. septicemia, diabetes mellitus, psychiatric disorders) increases according to corticosteroids dosage (21).

Nevertheless, type 2 diabetes mellitus could also precede the diagnosis of BP. In these cases, the link between these pathologies is not easy to understand, except for those patients previously treated with dipeptidyl peptidase-4 inhibitors (DPP-4 inhibitors), a quite recent class of hypoglycemic oral drugs frequently used in older diabetic patients and known to cause adverse skin reactions $(22,23)$. The mechanisms underlying the association of DPP-4 inhibitors with BP are still not clear. DPP-4 is an immune system's important enzyme, whose activity is altered in some autoimmune diseases, such as rheumatoid arthritis, systemic lupus erythematosus and inflammatory bowel diseases. Thus, in our study, the cases in which type 2 diabetes mellitus precedes the diagnosis of BP are difficult to interpret except for those patients (5/15) previously treated with DPP4inhibitors. Furthermore, our results are consistent with the
Table II. p-Values of each comorbidities observed in the sub-group of patients with BP (bullous pemphigoid). Bold values show significance.

\begin{tabular}{lc}
\hline Comorbidities in BP & $p$-Value \\
\hline Type 2 diabetes mellitus & $\mathbf{0 . 0 1 4 2}$ \\
Hypertension & 0.5453 \\
Cardiovascular diseases & 0.1040 \\
Neuropsychiatric disorders & $\mathbf{0 . 0 0 1 5}$ \\
Malignancies & 0.9000 \\
Autoimmune diseases & 0.6979 \\
Obstructive respiratory diseases & 1.0000 \\
Osteoporosis & 0.5436 \\
Kidney diseases & 0.2484 \\
\hline
\end{tabular}

most recent studies, according to which BP occurs more frequently in patients treated with vildagliptin, linagliptin and teneligliptin compared to other gliptins (24-28). Some studies suggest that BP associated with the use of DPP4inhibitors could be clinically and immunologically distinct from the conventional BP, due to the absence of the characteristic eosinophilic inflammatory infiltrate in the subepidermal blisters (25) and because of the frequent negativity of anti-BP180-NC16A antibodies (26).

Literature data about the association of BP with malignancies are controversial (29): the open question is whether there is an association between these pathologies and whether they share a common pathophysiologic mechanism. Among the different theories, one of the most accredited suggests that antibodies against tumor-specific antigens cross-react with antigens of epithelial basement membrane zone (BMZ) (30). Another hypothesis suggests that cancer cells could secrete hormone-like substances able to damage epithelial BMZ, resulting in the production of anti-basement membrane antibodies. Further theories consider the role of an external agent, such as a virus, which could be carcinogenic and, at the same time, able to damage the epithelial BMZ (31). Considering that BP predominantly 
affects the elderly, and that some recent reports suggest an increased risk for some solid tumors and lymphoproliferative disorders in BP, a higher frequency of malignant tumors was expected (31-33). However, other authors did not find a link between $\mathrm{PB}$ and malignancies (34). In our patients with BP, no association was found with malignant neoplasms $(8 / 102, p$-value $=0.9000)$. Therefore, we agree with those authors who recommend screening tests only when BP is characterized by severe systemic manifestations and/or atypical presentations (e.g. onset in young people).

Conversely, a statistically significant association was observed between BP and neuropsychiatric disorders ( $p$ value $=0.0015)$. The prevalence of neuropsychiatric pathologies in our BP patients was $19.6 \%$ (20/102). The most frequent neuropsychiatric disorders observed in our study were mood disorders (i.e. anxiety, depression) and psychosis (40\% of cases) followed by epilepsy (15\%), Parkinson's disease (10\%), Alzheimer's disease (10\%), strokes (10\%) and dementia, ALS and migraine with aura (15\%). Overall, our data are in agreement with the recent studies (35-38). The mean ages of patients with BP and neurological diseases and with BP without neurological comorbidities were 83.4 years and 75.9 years, respectively. The range of age between 80 and 89 years showed the highest association with neuropsychiatric diseases. In the literature, several studies have evaluated the association between mean age of patients with BP and neurological pathologies, showing that patients with neurological comorbidities were generally older than those without $(34,39,40)$. This result may be explained by the concept of immuno-senescence that is an age-related immune dysfunction with a consequent process of autoimmunization against antigens common between the CNS and epidermis (39). However, the chronological order of development of these two pathologies is unclear. The alteration of the blood-brain barrier in the course of neurological pathologies is a triggering factor for the development of an autoimmune response against antigens shared with BP $(41,42)$. However, the chronic inflammatory processes of BP acts like a promoter of neuronal degeneration $(43,44)$. Consequently, the relation between BP and neuropsychiatric disorders could be described as bidirectional.

The main limitation of this study is the small sample size of PV and PF which are rare pathologies characterized by a very low prevalence in the population. Nevertheless, we identified statistically significant associations of BP with type 2 diabetes mellitus and with neuropsychiatric diseases. The correlation with neuropsychiatric pathologies is interesting for the possible bidirectional role in their etiology. The association with type 2 diabetes mellitus could suggest more caution in the administration of systemic corticosteroids, especially in elderly patients.

\section{Conflicts of Interest}

The Authors declare that the research was conducted in the absence of any commercial or financial relationships that could be construed as a potential conflict of interest.

\section{Authors' Contributions}

MA: conception and design of the work. GG, MA and MF: data collection. MF, GTC and MA: drafting the article. All Authors read and approved the final manuscript.

\section{References}

1 Chen YJ, Wu CY, Lin MW, Chen TJ, Liao KK, Chen YC, Hwang CY, Chu SY, Chen CC, Lee DD, Chang YT, Wang WJ and Liu HN: Comorbidity profiles among patients with bullous pemphigoid: a nationwide population-based study. Brit J Dermatol 165: 593-599, 2011. PMID: 21517800. DOI: 10.1111/j.1365-2133.2011.10386.x

2 Hooten J, Hall R 3rd and Cardones A: Updates on the management of autoimmune blistering diseases. Skin Therapy Lett 19(5): 1-6, 2014. PMID: 25405675.

3 Heelan K, Mahar AL, Walsh S and Shear NH: Pemphigus and associated comorbidities: a cross-sectional study. Clin Exper Derm 40(6): 593-599, 2015. PMID: 25786337. DOI: 10.1111/ced.12634

4 Hsu DY, Brieva, J, Nardone B, West D and Silverberg JI: Association of pemphigus and systemic corticosteroid use with comorbid health disorders: a case-control study. Dermatol Online J 23(12): 1, 2017. PMID: 29447647.

5 Kibsgaard L, Bay B, Deleuran M and Vestergaard C: A retrospective consecutive case-series study on the effect of systemic treatment, length of admission time, and co-morbidities in 98 bullous pemphigoid patients admitted to a tertiary centre. Acta Derm Venereol 95: 307-311, 2015. PMID: 24979241. DOI: 10.2340/00015555-1925

6 Kremer N, Zeeli T, Sprecher E and Geller S: Failure of initial disease control in bullous pemphigoid: A retrospective study of hospitalized patients in a single tertiary center. Int J Dermatol 56(10): 1010-1016, 2017. PMID: 28856675. DOI: 10.1111/ ijd.13736

7 Lloyd-Lavery A, Chi C, Wojnarowska F and Taghipour K: The Associations Between Bullous Pemphigoid and Drug Use: A UK Case-Control Study. JAMA Dermatol 149(1): 58-62, 2013. PMID: 23324757. DOI: 10.1001/2013.jamadermatol.376

8 Ruocco V and Sacerdoti G: Pemphigus and bullous pemphigoid due to drugs. Int J Dermatol 30(5): 307-312, 1991. PMID: 1829722. DOI: $10.1111 / j .1365-4362.1991 . t b 03867 . x$

9 Ungprasert P, Wijarnpreecha K, and Thongprayoon C: Risk of venous thromboembolism in patients with bullous pemphigoid: A systematic review and meta-analysis. Indian J Dermatol Venereol Leprol 84: 22-26, 2018. PMID: 29185440. DOI: 10.4103/ijdvl.IJDVL_827_16

10 Aviña-Zubieta JA, Bhole VM, Amiri N, Sayre EC and Choi HK: The risk of deep venous thrombosis and pulmonary embolism in giant cell arteritis: A general population-based study. Ann Rheum Dis 75: 14854, 2016. PMID: 25265937. DOI: 10.1136/ annrheumdis-2014-205665. 
11 Bazzan M, Vaccarino A and Marletto F: Systemic lupus erythematosus and thrombosis. Thromb J 13: 16, 2015. PMID: 25908929. DOI: 10.1186/s12959-015-0043-3

12 Ungprasert P, Sanguankeo A, Upala S and Suksaranjit P: Psoriasis and risk of venous thromboembolism: A systematic review and meta-analysis. Q J Med 107: 793-797, 2014. PMID: 24713224. DOI: 10.1093/qjmed/hcu073

13 Ungprasert P, Srivali N, Spanuchart I, Thongprayoon C and Knight EL: Risk of venous thromboembolism in patients with rheumatoid arthritis: A systematic review and meta-analysis. Clin Rheumatol 33: 297-304, 2014. PMID: 24424839. DOI: 10.1007/s10067-014-2492-7

14 Ramagopalan SV, Wotton CJ, Handel AE, Yeates D and Goldacre MJ: Risk of venous thromboembolism in people admitted to hospital with selected immune-mediated diseases: Record-linkage study. BMC Med 9: 1, 2011. PMID: 21219637. DOI: 10.1186/1741-7015-9-1

15 Zöller B, Li X, Sundquist J and Sundquist K: Risk of pulmonary embolism in patients with autoimmune disorders: A nationwide follow-up study from Sweden. Lancet 379: 244-249, 2012. PMID: 22119579. DOI: 10.1016/S0140-6736(11)61306-8

16 Nagareddy P and Smyth SS: Inflammation and thrombosis in cardiovascular disease. Curr Opin Hematol 20: 457-463, 2013. PMID: 23892572. DOI: 10.1097/MOH.0b013e328364219d.

$17 \mathrm{Xu} \mathrm{J}$, Lupu F and Esmon CT: Inflammation, innate immunity and blood coagulation. Hamostaseologie 30: 5-6, 2010. PMID: 20162248

18 Cugno M, Tedeschi A, Borghi A, Bucciarelli P, Asero R, Venegoni L, Griffini S, Grovetti E, Berti E and Marzano AV: Activation of blood coagulation in two prototypic autoimmune skin diseases: a possible link with thrombotic risk. Plos One 10(6): e0129456, 2015. PMID: 26057532. DOI: 10.1371/journal.pone.0129456

19 Levi $\mathrm{M}$ and Van der Poll T: Two-way interactions between inflammation and coagulation. Trends Cardiovasc Med 15(7): 254259, 2005. PMID: 16226680. DOI: 10.1016/j.tcm.2005.07.004

20 Teixeira VB, Cabral R, Brites MM, Vieira R and Figueiredo A: Bullous pemphigoid and comorbidities: a case-control study in Portuguese patients. An Bras Dermatol 89(2): 274-278, 2014. PMID: 24770504. DOI: 10.1590/abd1806-4841.20142516

21 Joly P, Roujeau JC, Benichou J, Picard C, Dreno B, Delaporte E, Vaillant L, D'Incan M, Plantin P, Bedane C, Young P and Bernard P: A comparison of oral and topical corticosteroids in patients with bullous pemphigoid. N Engl J Med 346: 321-327, 2002. PMID: 11821508. DOI: 10.1056/NEJMoa011592

22 Itou M, Kawaguchi T, Taniguchi E and Sata M: Dipeptidyl peptidase4: a key player in chronic liver disease. World J Gastroenterol 19: 2298-2306, 2013. PMID: 23613622. DOI: 10.3748/wjg.v19.i15.2298

23 Kubota A, Maeda H, Kanamori A, Matoba K, Jin Y, Minagawa F, Obana M, Iemitsu K, Ito S, Amamiya H Kaneshiro M, Takai M, Kaneshige H, Hoshino K, Ishikawa M, Minami N, Takuma T, Sasai N, Aoyagi S, Kawata T, Mokubo A, Takeda H, Honda S, Machimura H, Motomiya T, Waseda M, Naka Y, Tanaka Y, Terauchi Y and Matsuba I: Efficacy and safety of sitagliptin monotherapy and combination therapy in Japanese type 2 diabetes patients. J Diabetes Investig 3: 503-509, 2012. PMID: 24843615. DOI: 10.1111/j.2040-1124.2012.00221.x

24 Béné J, Moulis G, Bennani I, Auffret M, Coupe P, Badai S, Hillaire-Buys D, Micallef J and Gautier S: Bullous pemphigoid and dipeptidyl peptidase IV inhibitors: a case-noncase study in the French Pharmacovigilance Database. Br J Dermatol 175: 296-301, 2016. PMID: 27031194. DOI: 10.1111/bjd.14601

25 Mai Y, Nishie W, Sato K, Hotta M, Izumi K, Ito K, Hosokawa $\mathrm{K}$ and Shimizu H: Bullous pemphigoid triggered by thermal burn under medication with a dipeptidyl peptidase-IV inhibitor: A case report and review of the literature. Front Immunol 9: 542, 2018. PMID: 29706950. DOI: 10.3389/fimmu.2018.00542

26 Murakami T, Yabe D and Inagaki N: Bullous pemphigoid with dipeptidyl peptidase-4 inhibitors: Clinical features and pathophysiology. J Diabetes Investig 10(5): 1168-1170, 2019. PMID: 30989811. DOI: 10.1111/jdi.13060

27 Kawaguchi Y, Shimauchi R, Nishibori N, Kawashima K, Oshitani S, Fujiya A, Shibata T, Ohashi N, Izumi K, Nishie W, Shimizu H, Arima H and Sobajima H: Dipeptidyl peptidase-4 inhibitors-associated bullous pemphigoid: A retrospective study of 168 pemphigoid and 9,304 diabetes mellitus patients. J Diabetes Investig 10(2): 392-398, 2019. PMID: 29920976. DOI: $10.1111 /$ jdi.12877

28 Lindgren O, Varpuluoma O, Tuusa J, Ilonen J, Huilaja L, Kokkonen $\mathrm{N}$ and Tasanen K: Gliptin-associated bullous pemphigoid and the expression of dipeptidyl peptidase-4/CD26 in bullous pemphigoid. Acta Derm Venereol 99: 602-609, 2019. PMID: 30848289. DOI: 10.2340/00015555-3166

29 Kalińska-Bienias A, Kowalczyk E, Jagielski P, Bienias P, Kowalewski $\mathrm{C}$ and Woźniak K: The association between neurological diseases, malignancies and cardiovascular comorbidities among patients with bullous pemphigoid: Casecontrol study in a specialized Polish center. Adv Clin Exp Med 28(5), 2019. PMID: 30784240. DOI: 10.17219/acem/90922

30 Balestri R, Magnano M, La Placa M, Patrizi A, Angileri L, Tengattini and Bardazzi F: Malignancies in bullous pemphigoid: A controversial association. J Dermatol 43(2): 125-133, 2016. DOI: 10.1111/1346-8138.13079

31 Venning VA and Wojnarowska F: The association of bullous pemphigoid and malignant disease: a case control study. Br J Dermatol 123: 439-445, 1990. PMID: 2095174. DOI: 10.1111/j.1365-2133.1990.tb01447.x

32 Ogawa H, Sakuma M, Morioka S, Kitamura K, Sasai S, Imamura $\mathrm{S}$ and Inaba Y: The incidence of internal malignancies in pemphigus and bullous pemphigoid in Japan. J Dermatol Sci 9: 136-141, 1995. PMID: 7772576. DOI: 10.1016/0923-1811 (94)00371-k

33 Yanagi T, Kato N, Yamane N and Osawa R: Bullous pemphigoid associated with dermatomyositis and colon carcinoma. Clin Exp Dermatol 32: 291-294, 2007. PMID: 17397352. DOI:10.1111/ j.1365-2230.2007.02368.x

34 Jedlickova H, Hlubinka M, Pavlik T, Semradova V, Budinska E and Vlasin Z: Bullous pemphigoid and internal diseases - A case-control study. Eur J Dermatol 20: 96-101, 2010. PMID: 19797038. DOI: 10.1684/ejd.2010.0805

35 Brick KE, Weaver CH, Savica R, Lohse CM, Pittelkow MR, Boeve $\mathrm{BF}$, Gibson LE, Camilleri MJ and Wieland CN: A population-based study of the association between bullous pemphigoid and neurologic disorders. J Am Acad Dermatol 71(6): 1191-1197, 2014. PMID: 25174542. DOI: 10.1016/j.jaad.2014.07.052

36 Taghipour K, Chi CC, Vincent A, Groves RW, Venning V and Wojnarowska F: The association of bullous pemphigoid with cerebrovascular disease and dementia: A case-control study. Arch Dermatol 146(11): 1251-1254, 2010. PMID: 21079062. DOI: 10.1001/archdermatol.2010.322 
37 Langer-Gould A, Albers KB, Van Den Eeden SK and Nelson LM: Autoimmune diseases prior to the diagnosis of multiple sclerosis: a population-based case-control study. Mult Scler 16: 855-861, 2010. PMID: 20463037. DOI: 10.1177/1352458510369146

38 Pietkiewicz P, Gornowicz-Porowska J, Bowszyc-Dmochowska M, Bartkiewicz $\mathrm{P}$ and Dmochowski M: Bullous pemphigoid and neurodegenerative diseases: A study in a setting of a Central European university dermatology department. Aging Clin Exp Res 28(4): 659-663, 2016. PMID: 26420424. DOI: 10.1007/s40520015-0459-4

39 Gambichler T, Segert H, Höxtermann S, Schmitz L, Altmeyer P and Teegen B: Neurological disorders in patients with bullous pemphigoid: clinical and experimental investigations. J Eur Acad Dermatol 29: 1758-1762, 2015. PMID: 25651418. DOI: 10.1111/ jdv.12995

40 Daneshpazhooh M, Khorassani J, Balighi K, Gandhi N, Mahmoudi H, Tohidinik H, Hemzelou S and Chams-Davatchi C: Neurological diseases and bullous pemphigoid: A case-control study in Iranian patients. Indian J Dermatol Venereol Leprol 83(2): 195-199, 2017. PMID: 27679408. DOI: 10.4103/03786323.191132
41 Seppänen A: Collagen XVII: a shared antigen in neurodermatological interactions? Clin Dev Immunol 2013: 240570, 2013. PMID: 23878581. DOI: $10.1155 / 2013 / 240570$

42 Chen J, Li L, Chen J, Zeng Y, Xu H, Song Y and Wang B: Sera of elderly bullous pemphigoid patients with associated neurological diseases recognize bullous pemphigoid antigens in the human brain. J Gerontol 57: 211-216, 2011. PMID: 20664178. DOI: $10.1159 / 000315393$

43 Bhattacharya A, Derecki NC, Lovenberg TW and Drevets WC: Role of neuro-immunological factors in the pathophysiology of mood disorders. Psychopharmacology (Berl) 233: 1623-1636, 2016. PMID: 26803500. DOI: 10.1007/s00213-016-4214-0

44 Naaldijk YM, Bittencourt MC, Sack U and Ulrich H: Kinins and microglial responses in bipolar disorder: a neuroinflammation hypothesis. Biol Chem 397: 283-296, 2016. PMID: 26859499. DOI: $10.1515 / \mathrm{hsz}-2015-0257$

Received March 23, 2020

Revised April 3, 2020

Accepted April 6, 2020 